\title{
Solid-Pseudopapillary Tumor of the Pancreas in Adults: A Case Report
}

\author{
Kamil Gulpinar ${ }^{1 *}$, Suleyman Ozdemir ${ }^{2}$, Erpulat Ozis ${ }^{2}$, Sadik Ersoz ${ }^{3}$ \\ ${ }^{1}$ Ufuk Universitesi Tip Fakultesi, Ankara, Turkey \\ ${ }^{2}$ Department of General Surgery, Ufuk University School of Medicine, Ankara, Turkey \\ ${ }^{3}$ Department of General Surgery, Ankara University School of Medicine, Ankara, Turkey \\ Email: *kgulpinar@yahoo.com
}

Received October 30, 2012; accepted November 30, 2012; accepted December 8, 2012

\begin{abstract}
Solid-pseudopapillary tumor of the pancreas (SPTP) is an uncommon low grade exocrine pancreatic malignancy. We represented a 22 years old female with an abdominal mass of 4 years history and symptoms of weakness, loss of appetite, 7 kilograms weight loss and swelling for almost 2 months. She was suspected of pancreatic malignancy due her abdominal ultrasound and CT reports and therefore underwent explorative surgery that revealed a huge pancreatic tumor .The tumor was resected totally and hystopathological examination reported significant components of pancreatic solid pseudopapiller tumor features which was also the final diagnosis.
\end{abstract}

Keywords: Solid-Pseudopapillary Tumor; Pancreas

\section{Introduction}

Solid-pseudopapillary tumor of the pancreas (SPTP) is an uncommon low grade exocrine pancreatic malignancy, mostly seen in young women with an incidence of $1 \%$ of all neoplasms of the exocrine pancreas. There had been many hypotheses about the histogenesis of the tumor. The most commonly agreed upon theory propose the origin of tumor to be derived from a pluripotential epithelial stem cell from small pancreatic ducts with exocrine ductal differentiation [1]. Additionally, the elevated progesterone receptors in the absence of estrogen receptors in these tumors seem to explain the prevalence in reproductive age women. The body and the tail of the pancreas are more frequently affected. The clinical presentation of the tumor is usually nonspecific and its diagnosis is usually incidental during diagnostic tests or surgical explorations. As the tumor is usually asymptomatic, the diagnosis is difficult and incidental during diagnostic tests and surgical explorations.

The aim of the current report is to present a case of this rare disease that diagnosed and treated in our clinic.

\section{Case Report}

A 22-year-old woman admitted to our clinic with one and a half month history of abdominal pain in the right upper quadrant. She also had corresponding complaints of

\footnotetext{
"Corresponding author.
}

weakness, loss of appetite, 7 kilograms weight loss and swelling. She claimed that a cystic lesion of the liver was detected by an abdominal ultrasonography 4 years ago and was interpreted as a benign cyst with no further evaluation.

At initial admission to our center, she brought her abdominal ultrasound report performed by another clinic one month ago showing a $7 \mathrm{~cm}$ diameter low echoic or iso-echoic round liver mass, which was heterogeneous, composed of solid and cystic components. Intrahepatic bile ducts and related vascular structures were all found to be normal in this report. In addition, she had had a CT examination in the same clinic and the mass were interpreted as (the patient only had the written report not the CT images) a liver mass located in left lobe medial segment with exophytic protrusions through the pancreas.

She had tenderness on the right upper quadrant in her physical examination and the liver was found to be palpable $3 \mathrm{~cm}$ below the 12th costa. Other systemic examinations were all normal.

Her laboratory values including tumor markers: alphafetoprotein, carcinoembryonic antigen, CA-125, CA15-3, CA19-9 were found to be within normal limits except for high erythrocyte sedimentation rate of $66 \mathrm{~mm} / \mathrm{h}$ (normal range: 0 - 20) and CRP: 17, 18 (normal range: 0 - 5).

Abdominal ultrasonography revealed a heterogeneous, iso-echoic tumor composed of solid and cystic components. She underwent an abdominal CT examination that 
revealed a $9 \times 8 \times 7 \mathrm{~cm}$ heterogenous cystic-solid mass localized at the distal pancreas including the tail and corpus, protruding through liver left lobe medial segment, that has hypodense necrotic spaces which enhanced peripherally after intravenous contrast injection (Figure 1). The bile duct and pancreatic duct showed significant dilatation. The margins of the tumor and its relation with pancreatic tissue and other organs couldn't be distinguished by CT images as the mass was huge comprising left liver lobe medial segment and the pancreas. The mass revealed a significant vascularization during the arterial phase of the CT. The CT findings were concordant with pancreatic carcinoma or SPT and interpreted accordingly.

The upper GIS endoscopy examination was normal except for an external gastric corpus and antrum compression from the direction of small curvature.

An angio-CT were also performed and revealed a huge avascular or hypovascular pancreatic mass.

Preoperative diagnosis was decided to be a pancreatic carcinoma and explorative surgery was planned. During the surgical exploration; a heterogeneous, irregular approximately $10 \mathrm{~cm}$ diameter pancreatic mass originating from uncinate process of the pancreas was observed. The tumor was ascending on the duodenum through the choleduct, hemi circulating on the anterior wall of duodenum with no invasion. The tumor seemed to repress the pancreatic head and tail below the duodenum and was lateralizing the stomach. In addition, invasion of the proximal choleduct above the duodenum was also present. The invasion through the choleduct was recognized while the mass tried to be dissected below the duodenumwith its capsule. As the tumor was originated from

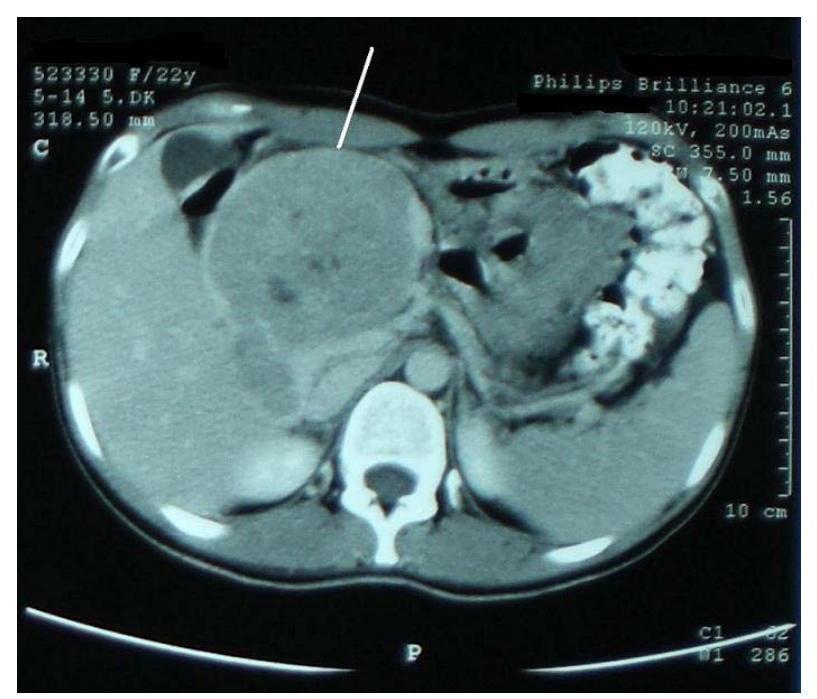

Figure 1. Abdominal contrasted-CT; Heterogenous cysticsolid like mass localized at the distal pancreas that has hypodense necrotic spaces which enhanced peripherally after intravenous contrast. uncinate process a total excision including uncinate process of pancreas was performed. As the proximal choleduct was invaded, a choledochotomy followed by a choledocoduedonostomy was also performed with a feedingtube replaced within. The mass was completely resected without any complications during the surgery.

The postoperative course was uneventful and no adjuvant therapy was given.

The patient was discharged from the hospital on postoperative 15 th day.

The pathological investigation showed a $90 \times 85 \times 95$ cm pancreatic tumor partially demarcated by a fibrous capsule. Heterogeneous solid pseudopapiller regions had areas of extensive necrosis and hemorrhagic material.

Microscopic hystopathological examination demonstrated that the cells that form solid and papillary regions were uniform without significant atypia and very few mitotic activities were observed. The hystopathological findings concluded the diagnosis as pancreatic solid pseudopapiller tumor (solid-cystic papillary neoplasm; Gruber Frantz Tumor) (Figure 2).

\section{Discussion}

Solid-pseudopapillary tumor of the pancreas (SPTP) is an uncommon low grade exocrine pancreatic malignancy that rarely metastases and named after Frantz VK who first reported the disease as "papillary tumor of the pancreas, benign or malignant” [2]. The body and the tail of the pancreas are more frequently affected (64\%) than the head [3]. But these huge tumors may repress other parts of the pancreas concluding with atrophy. Unlike the most previous reports our patient had SPTP originating from the head of the pancreas and specifically unicinate process of the head which is very rare.

Review of the literature by different authors reported

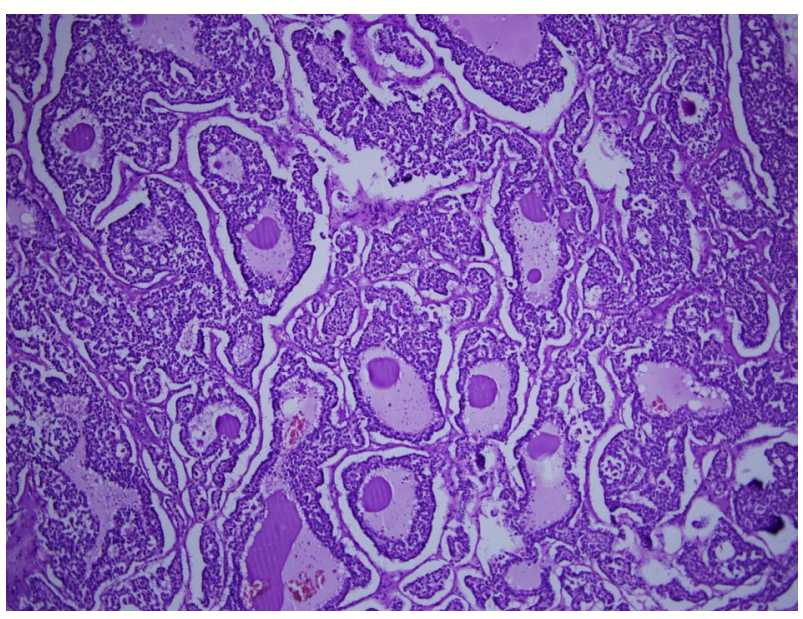

Figure 2. Hystopathological examination: cells that form solid and papillary regions that is uniform without significant atypia and very few mitotic activities. 
452 [4] and 718 [5] cases of SPT. In the last decade there had been an apparent increase in the number of diagnosed cases with the alertness of the disease by accumulating cases. The incidence of the disease is reported to be $1 \%-2 \%$ of all pancreatic tumors occurring in young females in their second or third decade of life [4-6]. The incidence of overall male occurrences were reported to be only $6.6 \%$ [4] and 3.9\% [5], respectively.

These tumors distinguish from other exocrine pancreatic tumors by particular pathological characteristics. Our patient total tumor size was approximately $10 \mathrm{~cm}$ as the reported size of these tumors vary widely between 0.5 $\mathrm{cm}$ through $25 \mathrm{~cm}$ with a mean of $9.3 \mathrm{~cm}$ [4]. In general they are big, round, well circumscribed with a pseudocapsule, full of mucinous secretion, and multilocular at times [7]. Microscopically, cysts are made up of an epithelial column (cystadenomas) or a combination of epithelial column and atypical epithelial cells (cystadenocarcinomas) [1]. The solid regions composed of nests and sheets of uniform epiteloid cells alternating with cystic spaces and pseudopapillae. The cells are rather discohesive with cytoplasmic delicacy and nuclear membrane irregularities. Pseudopapillaes formed by tumor cells around central vessels are commonly seen. Pleomorphism and mitotic figures of the tumor cells are typically absent. The tumor with its pseudocapsule usually displaces but does not invade adjacent structures [8,9]. The hystopathogenetic evaluation of our patients' tumor showed typical findings as described above with heterogeneous solid pseudopapiller regions with extensive necrosis and hemorrhagic material. The cells were uniform without significant atypia and very few mitotic activities were observed. It is challenging to make an accurate differential diagnosis from other solid and pancreatic lesions like inflammatory pseudocysts, mucinous cystic tumors, cystadenocarcinomas, pancreoblastoma, etc. But young age, gender and clinical course might help. However hepatic tumors should also be considered in differential diagnosis. Our patient was thought to have a benign cystic liver CT shown lesion 4 years before admission and the tumors features observed during preoperative diagnosis supported a liver mass, as well.

Immunohistochemistry evaluations of the pancreatic tumor in the diagnostic process of STPD s are somewhat diverse and no specific marker is yet reported. The tumor was shown to express variable cytokeratin, vimentin, and alpha1-antitrypsin positivity. Some neuroendocrine markers were also studied and synaptophysin and chromogranin A were shown to be expressed in these tumors with low specifity. CD56 and CD10 were also studied and reported to be valuable in the differential diagnosis of pancreatic endocrine tumor $[10,11]$. The tumor markers (CA19-9, CA242, CA50, CA72-2) have diagnostic value in malignant pancreatic cancers but not in SPTDs
[12]. Our patient laboratory evaluations were concordant with these findings as no elevations of tumor markers were present. The high sedimentation rate was non-specific and was not helpful during the diagnostic process.

The main preoperative problem still seems to be the misdiagnosis of an SCPT. However diffuse abdominal discomfort or vague pain in the epigastrium or left hypochondria is almost always present during the course of the disease for a number of years before the diagnosis. The abdominal pain or discomfort may be acute as was in our patient with only a 1.5 month history. Uncommonly seen weight loss of $7 \mathrm{~kg}$ per 2 months was one of the most significant symptoms of her disease suggesting a malign behavior tumor. Acute manifestations such as pancreatitis triggered by ischemia, distention or duct obstruction, or hemoperitoneum caused by the rupture of the tumor capsule are also rare [13,14]. A palpable abdominal mass in the upper abdomen or bile duct compression symptoms when the tumor is located at the head of pancreas are rare but should be kept in mind. Unfortunately, there is no specific physical examination finding of the disease as we were also confused with the enlargement of the liver during her physical examination and the palpated mass imitated and suggested a liver tumor at initial visit.

In the diagnostic process, ultrasound is the preliminary imaging method which reveals a well-encapsulated, homogenous or heterogeneous solid-cystic mass [15]. In our patients abdominal USG, the mass reported to be located at liver left lobe medial segment with protrusions through the pancreas and duodenum.

CT scans are also useful of showing a well circumscribed retroperitoneal mass with various solid areas and calcifications around hyper dense cystic components owing bleeding areas and hemorrhagic degeneration [16]. We performed CT and angio-CT imaging techniques which showed those typical findings of pancreatic tumor or SPTD appearances with good localization of the origin and was very helpful in our diagnosis.

MRI provides similar findings but reported to be more informative for differentiating certain tissue characteristics like hemorrhage, cystic degeneration or the presence of capsule [17].

Preoperative endoscopic ultrasonography-guided fine needle aspiration biopsy for preoperative detection of tumor are supported by some authors as imaging techniques are sometimes confusing due to similarity of findings among cystic lesions of pancreas [18]. In controversy this might be unacceptable by some because of uncertainty in diagnosis and the possible spread of tumor cells $[18,19]$.

Tumor resectability consideration preoperatively is often provided by improved imaging techniques that offers a more accurate extension diagnosis. Vascular infil- 
tration is the main factor for non-resectability. The sensitivity of helical CT for a successful assessment of such vascular infiltration is reported to be only $60 \%-89 \%$ causing undetected non-resectability. However volumetric angio-CT is superior by demonstrating the anatomic relation to the vascular tree, thus allowing a more appropriate planning for surgery [1].

SPT has a low grade malignant potential and has a very slow growing pattern. The doubling time of this malignancy were reported to be 677 days, 240 days and 765 days in different studies [20]. The first probable tumor of our patient as we informed in words, was observed incidentally 4 years before any symptomatic disease, unfortunately, we were not able to find the genuine USG report of that time. She also noted that, the incidentally observed tumor was misdiagnosed as a liver benign cyst with no further evaluation recommended.

Surgery is the only valid option with proven effectiveness for SPTP [21,22]. The accurate diagnosis of this low potential malignant tumor is important as the complete resection of the tumor reported to provide a $97 \%$ survival rate [23].

The surgical method depends on the size and location of the tumor and its relationships with the surrounding organs. A careful exploration during surgery should be done as to assess various arterial and venous vascular structures. STD s usually has a benign clinical course. Some challenging cases may present with local recurrence or distant metastases. In addition a locally advanced or metastatic disease at initial presentation is also possible in minority of the patients [20]. The common sites of metastasis include liver, peritoneum and lymph nodes. In addition it might invade adjacent tissues and vascular structures. In a review of 497 patients, 26 of them had (5\%) portal vein invasion and 44 of them had (9\%) other organ invasions (spleen, colon, duodenum, etc.) at the initial surgery [5].

Resection should be considered in case the upper mesenteric artery, hepatic artery and portal vein is observed to be tumor free. Well-encapsulated tumors, as reported here, usually enable simple enucleating of the neoplasm.

For advanced local invasive disease, to preserve the third and fourth duodenal portions, treitz ligament and first jejunal loop with careful dissection is extremely important [24]. For tumors of the pancreatic head, duedenopancreatectomy is suggested with recommendation of pylorus preservation for fewer digestive disturbances $[19,25]$. In our case, fortunately the tumor seem to be well-capsulated and originated from the unicinate process of the pancreatic head and simple resection of the unicinate process was enough with dissection of the tumor anterior to duodenum. As no local invasion to the duodenum were present, no resection of duodenal tissue was necessary for full resection. Only invasive focus was recognized on the choleduct and full excision of the invasive portion of the choleduct were performed with following choledochoduedonostomy. Body-tail pancreatectomy including splenectomy is indicated for tumors located in the body or tail of the gland. Depending on the low recurrence rate and prolonged survival after complete local resection, aggressive attempts at complete resection seem to be warranted, even if accompanied by metastases [21,26-28]. But neither extensive lymphatic dissection nor adjacent structure resection is reported to be reasonable [24].

Recurrence after apparently radical resection of a SPT can occur in $10 \%-15 \%$ of cases and liver is the common site [5,23,29-31]. Resection of these metastasis long term effects are still unpredictable but seem to improve survival rates [20]. The role of chemotherapy, radiotherapy and other medical treatment options are still under study with no proven efficiency of benefit or vice versa [32, 33].

\section{REFERENCES}

[1] M. H. Pascual and E. F. Herrero, "Solid-Cystic Papillary Epithelial Neoplasm of the Pancreas. A Diagnostic and Therapeutic Challenge,” Revista Española de Enfermedades Digestivas, Vol. 98, No. 11, 2006, pp. 801-808. doi:10.4321/S1130-01082006001100001

[2] V. K. Frantz, "Tumors of the Pancreas," Armed Forces Institute of Pathology, Washington DC, 1959, pp. 32-33.

[3] W. Rebhandl, F. X. Felberbauer, S. Puig, K. Paya, S. Hochschorner, M. Barlan and E. Horcher, "Solid-Pseudopapillary Tumor of the Pancreas (Frantz Tumor) in Children: Report of Four Cases and Review of the Literature," Journal of Surgical Oncology, Vol. 76, No. 4, 2001, pp. 289-296. doi:10.1002/jso.1048

[4] K. Y. Lam, C. Y. Lo and S. T. Fan, "Pancreatic SolidCystic-Papillary Tumor: Clinicopathologic Features in Eight Patients from Hong Kong and Review of the Literature,” World Journal of Surgery, Vol. 23, No. 10, 1999, pp. 1045-1050. doi:10.1007/s002689900621

[5] T. Papavramidis and S. Papavramidis, "Solid Pseudopapillary Tumors of the Pancreas: Review of 718 Patients Reported in English Literature,” Journal of the American College of Surgeons, Vol. 200, No. 6, 2005, pp. 965-972. doi:10.1016/j.jamcollsurg.2005.02.011

[6] D. C. Schwartz and M. A. Campos, "A Woman with Recurrent Abdominal Pain,” American Journal of the Medical Sciences, Vol. 321, No. 5, 2001, pp. 352-354. doi:10.1097/00000441-200105000-00009

[7] D. S. Klimstra, B. M. Wenig and C. S. Heffess, "SolidPseudopapillary Tumor of the Pancreas: A Typically Cystic Carcinoma of Low Malignant Potential,” Seminars in Diagnostic Pathology, Vol. 17, No. 1, 2000, pp. 66-80.

[8] T. Tornoczky, E. Kalman, P. Jakso, G. Mehes, L. Pajor, G. G. Kajtar, I. Battyany, S. Davidovics, M. Sohail and T. Krausz, "Solid and Papillary Epithelial Neoplasm Arising 
in Heterotopic Pancreatic Tissue of the Mesocolon,” Journal of Clinical Pathology, Vol. 54, No. 3, 2001, pp. 241-245. doi:10.1136/jcp.54.3.241

[9] G. Balercia, G. Zamboni, G. Bogina and G. M. Mariuzzi, "Solid-Cystic Tumor of the Pancreas. An Extensive Ultrastructural Study of Fourteen Cases," Journal of Submicroscopic Cytology and Pathology, Vol. 27, No. 3, 1995, pp. 331-340.

[10] K. Notohara, S. Hamazaki, C. Tsukayama, S. Nakamoto, K. Kawabata, K. Mizobuchi, K. Sakamoto and S. Okada, "Solid-Pseudopapillary Tumor of the Pancreas: Immunohistochemical Localization of Neuroendocrine Markers and CD10,” American Journal of Surgical Pathology, Vol. 24, No. 10, 2000, pp. 1361-1371. doi:10.1097/00000478-200010000-00005

[11] A. L. Adams, G. P. Siegal and N. C. Jhala, "Solid Pseudopapillary Tumor of the Pancreas: A Review of Salient Clinical and Pathologic Features,” Advances in Anatomic Pathology, Vol. 15, No. 1, 2008, pp. 39-45. doi:10.1097/PAP.0b013e31815e5237

[12] E. Karagulle, E. Yildirim, E. Turk, H. Kiyici and H. Karakayali, "Solid Pseudopapillary Tumor of the Pancreas: A Case Report,” Turkish Journal of Gastroenterology, Vol. 17, No. 4, 2006, pp. 316-319.

[13] J. Sakagami, K. Kataoka, Y. Sogame, A. Taii, T. Ojima, D. Kanemitsu, R. Takada, R. Ito, T. Motoyoshi, H. Yasuda, et al., "Solid Pseudopapillary Tumor as a Possible Cause of Acute Pancreatitis," Journal of the Pancreas, Vol. 5, No. 5, 2004, pp. 348-352.

[14] H. Omori, H. Asahi, Y. Inoue, T. Irinoda and K. Saito, "Solid and Cystic Tumor of the Pancreas with Massive Hemoperitoneum,” Hepatogastroenterology, Vol. 52, No. 63, 2005, pp. 936-939.

[15] M. D’Onofrio, R. Malago, F. Vecchiato, G. Zamboni, M. Testoni, M. Falconi, P. Capelli and R. P. Mucelli, "Contrast-Enhanced Ultrasonography of Small Solid Pseudopapillary Tumors of the Pancreas: Enhancement Pattern and Pathologic Correlation of 2 Cases," Journal of Ultrasound in Medicine, Vol. 24, No. 6, 2005, pp. 849-854.

[16] F. Miao, Y. Zhan, X. Y. Wang, D. B. Wang, K. M. Chen, A. R. Tang and H. Jiang, "CT Manifestations and Features of Solid Cystic Tumors of the Pancreas," Hepatobiliary \& Pancreatic Diseases International, Vol. 1, No. 3, 2002, pp. 465-468.

[17] V. Cantisani, K. J. Mortele, A. Levy, J. N. Glickman, P. Ricci, R. Passariello, P. R. Ros and S. G. Silverman, "MR Imaging Features of Solid Pseudopapillary Tumor of the Pancreas in Adult and Pediatric Patients," American Journal of Roentgenology, Vol. 181, No. 2, 2003, pp. 395401.

[18] R. H. Bardales, B. Centeno, J. S. Mallery, R. Lai, M. Pochapin, G. Guiter and M. W. Stanley, "Endoscopic Ultrasound-Guided Fine-Needle Aspiration Cytology Diagnosis of Solid-Pseudopapillary Tumor of the Pancreas: A Rare Neoplasm of Elusive Origin but Characteristic Cytomorphologic Features,” American Journal of Clinical Pathology, Vol. 121, No. 5, 2004, pp. 654-662. doi:10.1309/DKK2B9V4N0W26A8Q

[19] A. Raffel, K. Cupisti, M. Krausch, S. Braunstein, B.
Trobs, P. E. Goretzki and U. Willnow, "Therapeutic Strategy of Papillary Cystic and Solid Neoplasm (PCSN): A Rare Non-Endocrine Tumor of the Pancreas in Children,” Surgical Oncology, Vol. 13, No. 1, 2004, pp. 1-6. doi:10.1016/j.suronc.2003.09.003

[20] C. Sperti, M. Berselli, C. Pasquali, D. Pastorelli and S. Pedrazzoli, “Aggressive Behaviour of Solid-Pseudopapillary Tumor of the Pancreas in Adults: A Case Report and Review of the Literature," World Journal of Gastroenterology, Vol. 14, No. 6, 2008, pp. 960-965. doi:10.3748/wig. 14.960

[21] I. Hassan, I. Celik, C. Nies, A. Zielke, B. Gerdes, R. Moll, A. Ramaswamy, H. J. Wagner and D. K. Bartsch, "Successful Treatment of Solid-Pseudopapillary Tumor of the Pancreas with Multiple Liver Metastases,” Pancreatology, Vol. 5, No. 2-3, 2005, pp. 289-294. doi:10.1159/000085285

[22] R. Salvia, C. Bassi, L. Festa, M. Falconi, S. Crippa, G. Butturini, A. Brighenti, P. Capelli and P. Pederzoli, "Clinical and Biological Behavior of Pancreatic Solid Pseudopapillary Tumors: Report on 31 Consecutive Patients,” Journal of Surgical Oncology, Vol. 95, No. 4, 2007, pp. 304-310. doi:10.1002/jso.20685

[23] A. K. Madan, C. B. Weldon, W. P. Long, D. Johnson and A. Raafat, "Solid and Papillary Epithelial Neoplasm of the Pancreas," Journal of Surgical Oncology, Vol. 85, No. 4, 2004, pp. 193-198. doi:10.1002/jso.20019

[24] S. Q. Chen, S. Q. Zou, Q. B. Dai and H. Li, "Clinical Analysis of Solid-Pseudopapillary Tumor of the Pancreas: Report of 15 Cases," Hepatobiliary \& Pancreatic Diseases International, Vol. 7, No. 2, 2008, pp. 196-200.

[25] C. A. Pellegrini, C. F. Heck, S. Raper and L. W. Way, "An Analysis of the Reduced Morbidity and Mortality Rates after Pancreaticoduodenectomy," Archives of Surgery, Vol. 124, No. 7, 1989, pp. 778-781. doi:10.1001/archsurg.1989.01410070028006

[26] D. T. Alexandrescu, K. O’Boyle, A. Feliz, A. Fueg and P. H. Wiernik, "Metastatic Solid-Pseudopapillary Tumour of the Pancreas: Clinico-Biological Correlates and Management," Clinical Oncology, Vol. 17, No. 5, 2005, pp. 358-363. doi:10.1016/j.clon.2004.11.015

[27] S. M. de Castro, D. Singhal, D. C. Aronson, O. R. Busch, T. M. van Gulik, H. Obertop and D. J. Gouma, "Management of Solid-Pseudopapillary Neoplasms of the Pancreas: A Comparison with Standard Pancreatic Neoplasms," World Journal of Surgery, Vol. 31, No. 5, 2007, pp. 1130-1135. doi:10.1007/s00268-006-0214-2

[28] S. Nagri, A. Abdu, S. Anand, M. Krishnaiah and V. Arya, "Liver Metastasis Four Years after Whipple’s Resection for Solid-Pseudopapillary Tumor of the Pancreas," Journal of the Pancreas, Vol. 8, No. 2, 2007, pp. 223-227.

[29] C. Mao, M. Guvendi, D. R. Domenico, K. Kim, N. R. Thomford and J. M. Howard, "Papillary Cystic and Solid Tumors of the Pancreas: A Pancreatic Embryonic Tumor? Studies of Three Cases and Cumulative Review of the World's Literature," Surgery, Vol. 118, No. 5, 1995, pp. 821-828. doi:10.1016/S0039-6060(05)80271-5

[30] A. Saiura, N. Umekita, Y. Matsui, T. Maeshiro, S. Miyamoto, M. Kitamura and A. Wakikawa, "Successful Sur- 
gical Resection of Solid Cystic Tumor of the Pancreas with Multiple Liver Metastases and a Tumor Thrombus in the Portal Vein," Hepatogastroenterology, Vol. 47, No. 33, 2000, pp. 887-889.

[31] L. H. Tang, H. Aydin, M. F. Brennan and D. S. Klimstra, "Clinically Aggressive Solid Pseudopapillary Tumors of the Pancreas: A Report of Two Cases with Components of Undifferentiated Carcinoma and a Comparative Clinicopathologic Analysis of 34 Conventional Cases," American Journal of Surgical Pathology, Vol. 29, No. 4, 2005, pp. 512-519. doi:10.1097/01.pas.0000155159.28530.88
[32] A. Maffuz, T. B. Fde, J. A. Silva and S. Torres-Vargas, "Preoperative Gemcitabine for Unresectable, Solid Pseudopapillary Tumour of the Pancreas," Lancet Oncology, Vol. 6, No. 3, 2005, pp. 185-186. doi:10.1016/S1470-2045(05)01770-5

[33] C. M. Kang, K. S. Kim, J. S. Choi, H. Kim, W. J. Lee and B. R. Kim, "Solid Pseudopapillary Tumor of the Pancreas Suggesting Malignant Potential,” Pancreas, Vol. 32, No. 3, 2006, pp. 276-280. doi:10.1097/01.mpa.0000202956.41106.8a 